\title{
Dielectric Relaxation of Benzonitrile in Benzene
}

\author{
Rajesh Kumar ${ }^{\mathrm{a}}$, Nagesh Thakur ${ }^{\mathrm{a}}$, and Rajendra S. Bisht ${ }^{\mathrm{b}}$ \\ ${ }^{a}$ Electronics Research Laboratory, Department of Physics, H. P. University, Shimla-171 005, \\ Himachal Pradesh, India \\ b TBRL, Chandigarh-160 030, India
}

Reprint requests to R. K.; E-mail: rajesh28kum@yahoo.co.in

Z. Naturforsch. 63a, 813 - 818 (2008); received May 13, 2008

The dielectric constant, $\varepsilon^{\prime}$, and dielectric loss, $\varepsilon^{\prime \prime}$, of dilute solutions of benzonitrile $\left(\mathrm{C}_{6} \mathrm{H}_{5} \mathrm{CN}\right)$ in benzene have been measured at $9.885 \mathrm{GHz}$ at $25,30,35$, and $40{ }^{\circ} \mathrm{C}$ using standard standing microwave techniques. Following the single frequency concentration variational method of Gopala Krishna, the dielectric relaxation time, $\tau$, and the dipole moment, $\mu$, at various temperatures have been calculated. It was concluded that dielectric relaxation processes can be treated as rate processes just like the viscous flow process. Based on the above studies, the monomer structure of benzonitrile in benzene has been inferred. The solute-solvent molecular association of benzonitrile in benzene has been found. The energy parameters $\Delta H_{\varepsilon}, \Delta F_{\varepsilon}, \Delta S_{\varepsilon}$ for the dielectric relaxation process of benzonitrile in benzene at different temperatures have been calculated and compared with the corresponding energy parameters $\Delta H_{\eta}, \Delta F_{\eta}, \Delta S_{\eta}$ for the viscous flow process.

Key words: Microwave Absorption; Dielectric Relaxation; Dielectric Constant; Benzonitrile; Solute-Solvent Interaction.

\section{Introduction}

Benzonitrile $\left(\mathrm{C}_{6} \mathrm{H}_{5} \mathrm{CN}\right)$ is a nonaqueous solvent which has a medium value of the dielectric constant $\left(\varepsilon^{\prime}=25.20\right)[1]$ and a high dipole moment $(\mu=$ $4.05 \mathrm{D})$ [1]. It is an excellent solvent for many anhydrous inorganic salts, organometallic compounds and a variety of organic substances [2]. Dielectric relaxation studies of polar molecules in a nonpolar solvent from microwave absorption data have been frequently attempted [3-9]. Dielectric relaxation studies in the microwave region provide meaningful information about various types of molecular associations [10], because microwaves allow to detect even weaker molecular interactions. This paper is concerned with dielectric relaxation studies of $\mathrm{C}_{6} \mathrm{H}_{5} \mathrm{CN}$ in benzene at different temperatures $\left(25,30,35\right.$, and $\left.40{ }^{\circ} \mathrm{C}\right)$ from microwave absorption measurements at $9.885 \mathrm{GHz}$. It is found that $\mathrm{C}_{6} \mathrm{H}_{5} \mathrm{CN}$ exists in monomer form in benzene solution. The solute-solvent molecular association is predicted. The energy parameters $\Delta H_{\varepsilon}, \Delta F_{\varepsilon}, \Delta S_{\varepsilon}$ for the dielectric relaxation process are calculated and compared with the corresponding energy parameters for the viscous flow process. It is found that the dielectric relaxation process is a rate process like the viscous flow process.

\section{Experimental}

Benzonitrile (GC grade; Merck, Germany) was distilled through a long vertical fractionating column under reduced pressure and the middle fraction was collected for use. Benzene (extra-pure AR; Sisco Research Laboratories Pvt. Ltd., Mumbai, India) was dried by refluxing over freshly cut sodium metal for 6 $8 \mathrm{~h}$ and then distilled through a long vertical fractionating column. The middle fraction of the distilled benzene was collected for use. The X-band microwave bench was used to measure the wavelength in the dielectric medium and the voltage standing wave ratio (VSWR), using a short-circuiting plunger. Microwave techniques of Heston et al. [11] were used to calculate the dielectric constant, $\varepsilon^{\prime}$, and the dielectric loss, $\varepsilon^{\prime \prime}$, of dilute solutions of $\mathrm{C}_{6} \mathrm{H}_{5} \mathrm{CN}$ in benzene at different temperatures. The circulation of thermostated water around the dielectric cell controlled the temperature of the solution within $\pm 0.05{ }^{\circ} \mathrm{C}$. The viscosity and the density of benzene at different temperatures were measured with an Ubbelohde viscometer and a pycnometer, respectively. Also the dielectric relaxation time, $\tau$, and the dipole moment, $\mu$, of $\mathrm{C}_{6} \mathrm{H}_{5} \mathrm{CN}$ in benzene have been calculated following the single frequency concentration variational method of Gopala Krishna [12]. The 
Eyring rate equations [13] have been used to calculate the energy parameters for a dielectric relaxation process and viscous flow process.

\section{Results and Discussion}

The dielectric constant, $\varepsilon^{\prime}$, and the dielectric loss, $\varepsilon^{\prime \prime}$, of the dilute solutions of $\mathrm{C}_{6} \mathrm{H}_{5} \mathrm{CN}$ in benzene at 25 , 30,35 , and $40^{\circ} \mathrm{C}$ have been calculated according to the method of Heston et al. [11] and are given in Table 1. The following equations have been used:

$$
\varepsilon^{\prime}=\left(\frac{\lambda_{0}}{\lambda_{\mathrm{c}}}\right)^{2}+\left(\frac{\lambda_{0}}{\lambda_{\mathrm{d}}}\right)^{2}
$$

and

$$
\varepsilon^{\prime \prime}=\frac{2}{\pi}\left(\frac{\lambda_{0}}{\lambda_{\mathrm{d}}}\right)^{2} \frac{\lambda_{\mathrm{g}}}{\lambda_{\mathrm{d}}}\left(\frac{\mathrm{d} \rho}{\mathrm{d} n}\right) .
$$

Here $\lambda_{0}, \lambda_{\mathrm{c}}, \lambda_{\mathrm{g}}$, and $\lambda_{\mathrm{d}}$ are the free space wavelength, the cut-off wavelength, the waveguide wavelength, and the wavelength in the waveguide filled with the solution, respectively. $\rho$ is the inverse of the VSWR and $\mathrm{d} \rho / \mathrm{d} n$ is the slope of $\rho$ versus $n$, where $n=1,2,3$ $\ldots \varepsilon^{\prime}$ and $\varepsilon^{\prime \prime}$ were reproducible within $\pm 0.5 \%$ and $\pm 3.8 \%$, respectively. The relaxation time, $\tau$, and dipole moment, $\mu$, as given in Table 1 have been calculated by following Gopala Krishna's single frequency concentration variational method [12].

Gopala Krishna's method makes use of Debye's theory of dielectric relaxation. The Debye equation for the complex permittivity of the dielectric medium as a function of frequency of the applied electric field [14] can be written as

$$
\frac{\varepsilon^{*}-1}{\varepsilon^{*}+2}=\frac{\varepsilon_{\infty}-1}{\varepsilon_{\infty}+2}+\frac{4 \pi N_{1} \mu^{2}}{9 k T} \frac{1}{1+j \omega \tau},
$$

where $N_{1}$ is the number of polar molecules per unit volume and $\varepsilon^{*}=\varepsilon^{\prime}-j \varepsilon^{\prime \prime}$ is the complex permittivity of the medium. $\varepsilon_{\infty}$ is the optical permittivity and $\omega$ is the angular frequency.

Separating the real and imaginary parts of both sides of (3) yields

$$
\begin{aligned}
\frac{\varepsilon^{\prime 2}+\varepsilon^{\prime}+\varepsilon^{\prime 2}-2}{\left(\varepsilon^{\prime}+2\right)^{2}+\varepsilon^{\prime \prime 2}} & =\frac{\varepsilon_{\infty}-1}{\varepsilon_{\infty}+2}+\frac{4 \pi N_{1} \mu^{2}}{9 k T} \cdot \frac{1}{1+\omega^{2} \tau^{2}}, \\
\frac{3 \varepsilon^{\prime \prime}}{\left(\varepsilon^{\prime}+2\right)^{2}+\varepsilon^{\prime \prime 2}} & =\frac{4 \pi N_{1} \mu^{2}}{9 k T} \cdot \frac{\omega \tau}{1+\omega^{2} \tau^{2}} .
\end{aligned}
$$

\begin{tabular}{|c|c|c|c|c|c|}
\hline $\begin{array}{c}T \\
\left({ }^{\circ} \mathrm{C}\right) \\
\end{array}$ & $\begin{array}{c}\text { Mass fraction of } \\
\mathrm{C}_{6} \mathrm{H}_{5} \mathrm{CN} \text { in benzene }\end{array}$ & $\begin{array}{c}\varepsilon^{\prime} \\
\pm 0.5 \% \\
\end{array}$ & $\begin{array}{c}\varepsilon^{\prime \prime} \\
\pm 3.8 \% \\
\end{array}$ & $\begin{array}{c}\tau\left(10^{-12} \mathrm{~s}\right) \\
\pm 3.4 \%\end{array}$ & $\begin{array}{l}\mu(\mathrm{D}) \\
\pm 2.7 \% \\
\end{array}$ \\
\hline 25 & $\begin{array}{l}0.0020 \\
0.0046 \\
0.0066 \\
0.0086 \\
0.0103\end{array}$ & $\begin{array}{l}2.346 \\
2.376 \\
2.402 \\
2.425 \\
2.446\end{array}$ & $\begin{array}{l}0.0125 \\
0.0308 \\
0.0422 \\
0.0565 \\
0.0671\end{array}$ & 8.383 & 3.722 \\
\hline 30 & $\begin{array}{l}0.0020 \\
0.0046 \\
0.0066 \\
0.0086 \\
0.0103\end{array}$ & $\begin{array}{l}2.332 \\
2.366 \\
2.391 \\
2.413 \\
2.438\end{array}$ & $\begin{array}{l}0.0114 \\
0.0300 \\
0.0413 \\
0.0554 \\
0.0658\end{array}$ & 7.960 & 3.814 \\
\hline 35 & $\begin{array}{l}0.0020 \\
0.0046 \\
0.0066 \\
0.0086 \\
0.0103\end{array}$ & $\begin{array}{l}2.320 \\
2.353 \\
2.382 \\
2.405 \\
2.428\end{array}$ & $\begin{array}{l}0.0106 \\
0.0291 \\
0.0402 \\
0.0547 \\
0.0632\end{array}$ & 7.534 & 3.894 \\
\hline 40 & $\begin{array}{l}0.0020 \\
0.0046 \\
0.0066 \\
0.0086 \\
0.0103\end{array}$ & $\begin{array}{l}2.308 \\
2.341 \\
2.367 \\
2.394 \\
2.420\end{array}$ & $\begin{array}{l}0.0099 \\
0.0282 \\
0.0391 \\
0.0531 \\
0.0611\end{array}$ & 7.089 & 3.969 \\
\hline
\end{tabular}

Table 1. Dielectric constant, $\varepsilon^{\prime}$, dielectric loss, $\varepsilon^{\prime \prime}$, relaxation time, $\tau$, and dipole moment, $\mu$, of benzonitrile in benzene at different temperatures.

Putting

$$
\begin{aligned}
& X=\frac{\varepsilon^{\prime 2}+\varepsilon^{\prime}+\varepsilon^{\prime 2}-2}{\left(\varepsilon^{\prime}+2\right)^{2}+\varepsilon^{\prime 2}}, \\
& Y=\frac{3 \varepsilon^{\prime \prime}}{\left(\varepsilon^{\prime}+2\right)^{2}+\varepsilon^{\prime \prime 2}},
\end{aligned}
$$

and

$$
P=\frac{\varepsilon_{\infty}-1}{\varepsilon_{\infty}+2}
$$

in the above equations, one gets

$$
X=P+\frac{Y}{\varepsilon \tau} .
$$

The value of $P$ in (6) slightly varies over the concentration range of the dilute solutions. But as the variation of $\varepsilon^{\prime}$ and $\varepsilon^{\prime \prime}$ is far higher than the variation of $P$ due to the change in concentration of the dilute solutions at microwave frequencies, it can be treated as constant over the range of the concentration variation. From the slope of the curve $Y$ versus $X$ the value of the relaxation time of polar molecules in nonpolar solvents can be determined. It can be written as

$$
\tau=\frac{\lambda_{0}}{2 \pi c}\left(\frac{\mathrm{d} Y}{\mathrm{~d} X}\right) \text {. }
$$



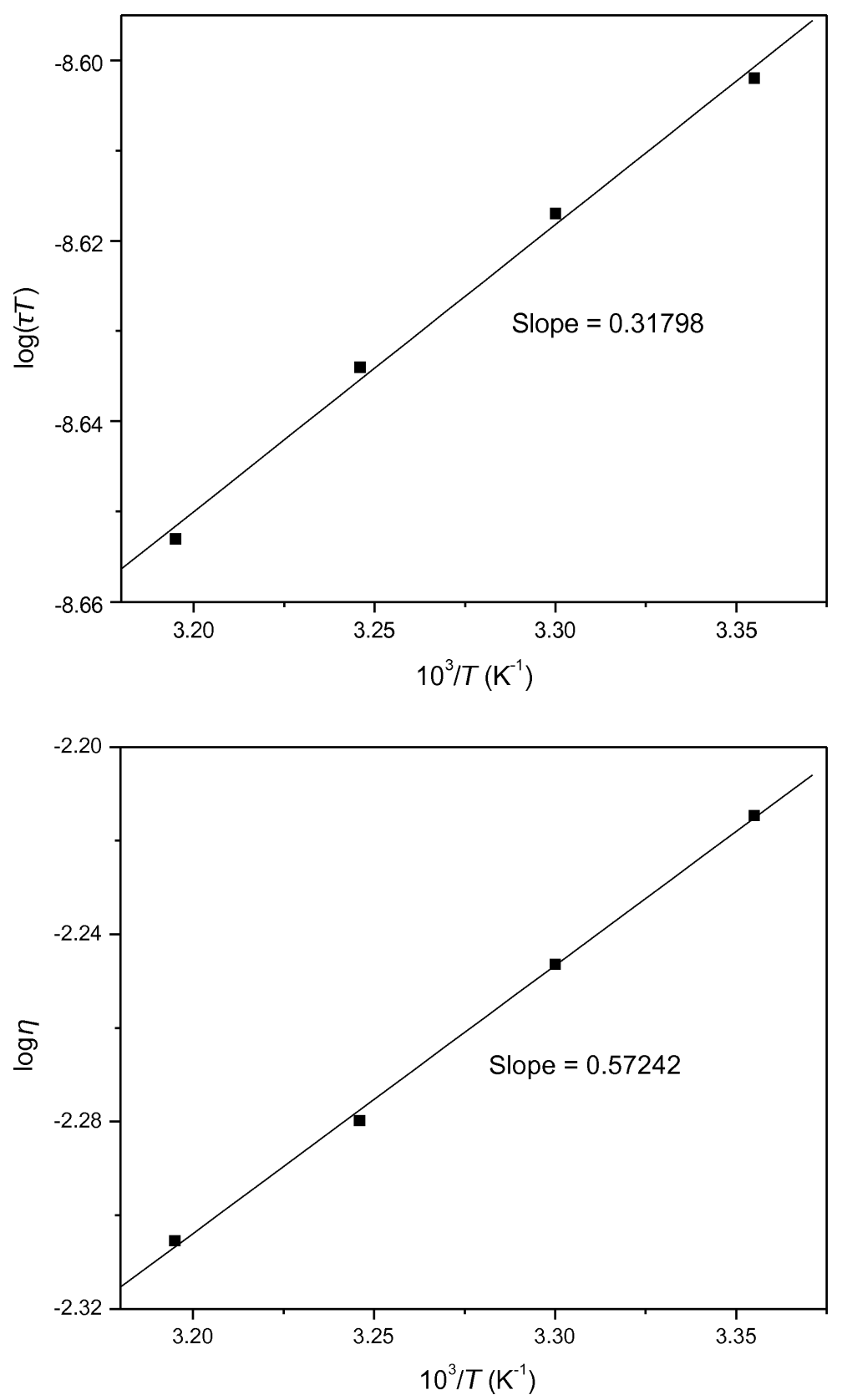

Fig. 1. Plot $\log (\tau T)$ versus $10^{3} / T$.

Fig. 2. Plot $\log \eta$ versus $10^{3} / T$.
To determine the dipole moment (6) can be written as

$$
X=P+K W d_{12}
$$

with

$$
K=\frac{4 \pi N \mu^{2}}{9 k T M\left(1+\omega^{2} \tau^{2}\right)}
$$

and

$$
N_{1}=\frac{N d_{12} W}{M},
$$

where $N$ is Avogadro's number, $M$ the molecular mass of the polar substance, $W$ the mass fraction, and $d_{12}$ the density of the solution. At low concentration the variation of the density of the solution $W$ may be taken as 
Table 2. Free energies of activation, $\Delta F_{\varepsilon}, \Delta F_{\eta}$, in $\mathrm{kJ} \mathrm{mol}^{-1}$, enthalpies of activation, $\Delta H_{\varepsilon}, \Delta H_{\eta}$, in $\mathrm{kJ} \mathrm{mol}^{-1}$, and entropies of activation, $\Delta S_{\varepsilon}, \Delta S_{\eta}$, in $\mathrm{J} \mathrm{mol}^{-1} \mathrm{~K}^{-1}$, of benzonitrile in benzene at different temperatures.

\begin{tabular}{ccccccc}
\hline$T$ & $\Delta F_{\varepsilon}$ & $\Delta H_{\varepsilon}$ & $\Delta S_{\varepsilon}$ & $\Delta F_{\eta}$ & $\Delta H_{\eta}$ & $\Delta S_{\eta}$ \\
$\left({ }^{\circ} \mathrm{C}\right)$ & $\pm 0.03 \%$ & $\pm 0.04 \%$ & $\pm 0.07 \%$ & & & \\
\hline 25 & 9.7975 & 6.0911 & -12.4375 & 12.1918 & 10.964 & -4.1194 \\
30 & 9.8731 & 6.0911 & -12.4818 & 12.2202 & 10.964 & -4.1453 \\
35 & 9.9371 & 6.0911 & -12.4869 & 12.2478 & 10.964 & -4.1674 \\
40 & 9.9818 & 6.0911 & -12.4304 & 12.3105 & 10.964 & -4.3012 \\
\hline
\end{tabular}

linear and is then given by the relation

$$
d_{12}=d_{0}(1+\alpha W),
$$

where $d_{0}$ is the density of the solvent. In the limited experimental range of concentration variation the graph between $X$ and $W$ can be taken as a straight line with the slope $\mathrm{d} X / \mathrm{d} W$ as $K d_{0}$. From this the value of the dipole moment can be calculated using the following relation:

$$
\mu^{2}=\frac{9 k T M}{4 \pi N d_{0}}\left[1+\left(\frac{\mathrm{d} Y}{\mathrm{~d} X}\right)^{2}\right] \frac{\mathrm{d} X}{\mathrm{~d} W} .
$$

The energy parameters for the dielectric relaxation process of $\mathrm{C}_{6} \mathrm{H}_{5} \mathrm{CN}$ in benzene and the corresponding energy parameters for the viscous flow of benzene, as given in Table 2, have been calculated using the following equations given by Eyring et al. [13] for the rate process:

$$
\begin{aligned}
& \tau=\frac{h}{k T} \exp \left(\Delta F_{\varepsilon} / R T\right), \\
& \Delta F_{\varepsilon}=\Delta H_{\varepsilon}-T \Delta S_{\varepsilon}, \\
& \eta=\frac{h N}{V} \exp \left(\Delta F_{\eta} / R T\right), \\
& \Delta F_{\eta}=\Delta H_{\eta}-T \Delta S_{\eta},
\end{aligned}
$$

where $\Delta F_{\varepsilon}, \Delta H_{\varepsilon}$, and $\Delta S_{\varepsilon}$ are the free energy, the enthalpy, and the entropy of activation for the dielectric relaxation process, and $\Delta F_{\eta}, \Delta H_{\eta}$, and $\Delta S_{\eta}$ are the corresponding energy parameters for the viscous flow process. $V$ is the molar volume of the solvent. The plots of $\log (\tau T)$ versus $10^{3} / T$ (Fig. 1) and $\log \eta$ versus $10^{3} / T$ (Fig. 2) according to (7) and (9) are found to be linear, which indicates that both the dielectric relaxation process and the viscous flow process can be treated as rate processes. The $\Delta H_{\varepsilon}$ and $\Delta H_{\eta}$ values are computed from the slope of the linear plot of $\log (\tau T)$ versus $10^{3} / T$ and $\log \eta$ versus $10^{3} / T$, respectively, using the relation: slope $=$ $\Delta H / 2.303 R$.
The variation of $\varepsilon^{\prime}$ and $\varepsilon^{\prime \prime}$ with the mass fraction of $\mathrm{C}_{6} \mathrm{H}_{5} \mathrm{CN}$ in benzene is found to be linear (Figs. 3 and 4). This shows that there is no change in the nature of the rotating molecular entities in the benzene solution. This ensures the applicability of Debye's theory and hence of Gopala Krishna's method for calculating the dielectric relaxation time and dipole moment of $\mathrm{C}_{6} \mathrm{H}_{5} \mathrm{CN}$ in a nonpolar solvent. It is observed that the dielectric relaxation time decreases with increasing temperature. This behaviour may be explained on the basis of Debye's theory of dielectric relaxation [15].

The dipole moment of $\mathrm{C}_{6} \mathrm{H}_{5} \mathrm{CN}$ in benzene is found to increase slightly with an increase in temperature and approaches the value known from the literature. This shows that $\mathrm{C}_{6} \mathrm{H}_{5} \mathrm{CN}$ exists in the monomer form in benzene. The change in the dipole moment with temperature may be due to the stretching of bond moments and the change in bond angles. The small variation of the value of the dipole moment with rising temperature may be attributed to the possible solute-solvent molecular association [16]. A solute-solvent molecular association for $\mathrm{C}_{6} \mathrm{H}_{5} \mathrm{CN}$ in benzene arises because of the interaction of the nitrile group of benzonitrile and the $\pi$-delocalized electron cloud of the benzene molecule (Fig. 5). The energy parameters $\Delta H_{\varepsilon}, \Delta F_{\varepsilon}, \Delta S_{\varepsilon}$ for the dielectric relaxation process and the energy parameters $\Delta H_{\eta}, \Delta F_{\eta}, \Delta S_{\eta}$ for the viscous flow process have been compared as shown in Table 2.

The free energy of activation $\Delta F_{\varepsilon}$ of the dielectric relaxation process is found to be less than the free energy of activation $\Delta F_{\eta}$ of the viscous flow process. This may be explained on the basis that the dielectric relaxation process includes only the rotation of the molecular complexes, whereas in the viscous flow process both rotational as well as the translation motion of the molecules are involved. It is found that the activation enthalpy $\Delta H_{\varepsilon}$ of the dielectric relaxation process is less than the activation enthalpy $\Delta H_{\eta}$ of the viscous flow process. The activation enthalpy depends on the local environment of the molecules. Different values for the activation enthalpy indicate that the dielectric relaxation process and the viscous flow process involve the breaking of bonds with the neighbouring molecules in a different way and to a different extent. The entropy of a system is a measure of the disorder of the system. According to Branin and Smyth [17], a negative value for the activation entropy indicates that a comparatively smaller number of configurations is possible in an activated state, which is more ordered in comparison to the normal state. This again indicates the presence of co- 

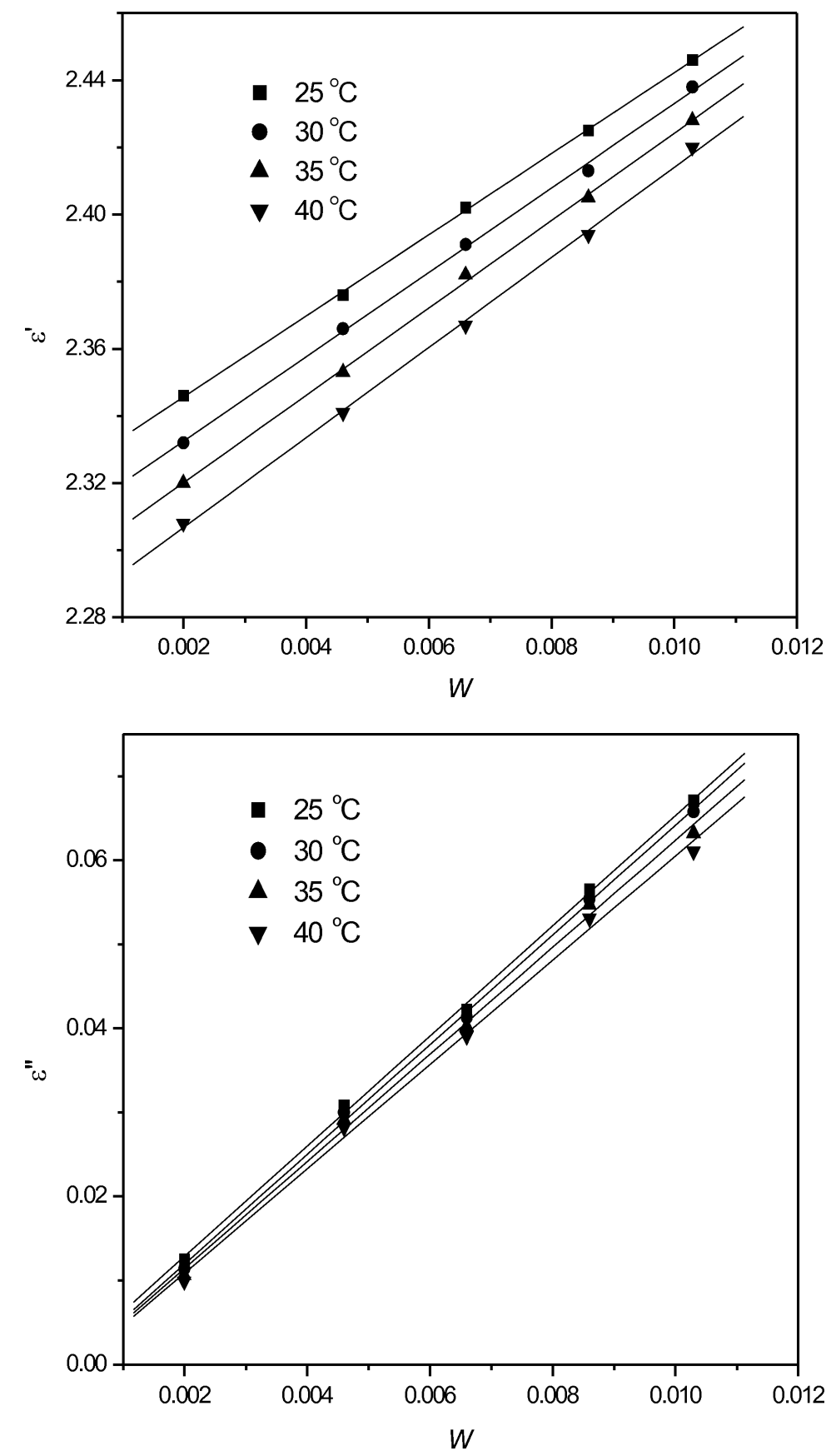

Fig. 3. Variation of the dielectric constant $\varepsilon^{\prime}$ with the mass fraction $W$ of benzonitrile in benzene at different temperatures.

Fig. 4. Variation of the dielectric loss $\varepsilon^{\prime \prime}$ with the mass fraction $W$ of benzonitrile in benzene at different temperatures.

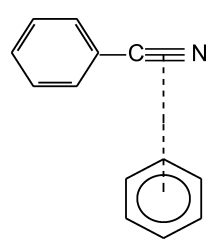

Fig. 5. Solute-solvent molecular association between benzonitrile and benzene. operative orientations of the molecules caused by the steric forces or strong dipole-dipole interactions with the dipoles being more aligned in the activated state. However, a positive activation entropy indicates that the activated state is more disordered. In the present case it is observed that the change in entropy of the 
dielectric relaxation process and the viscous flow process is negative, indicating that the activated state of both processes is more stable than the normal state.

\section{Conclusion}

The variation of the dipole moment, $\mu$, of $\mathrm{C}_{6} \mathrm{H}_{5} \mathrm{CN}$ in benzene shows that $\mathrm{C}_{6} \mathrm{H}_{5} \mathrm{CN}$ exists in the monomer form in benzene solution. This variation of the value

[1] A. Weissberger, E. S. Proskauer, J. A. Riddick, and E.E. Toops, Organic Solvents, 2nd ed., New York 1967, p. 230.

[2] A. K. Convington and T. Dickinson, Physical Chemistry of Organic Solvent Systems, Plenum Press, London and New York 1973, p. 12.

[3] V. Sharma, N. Thakur, D. R. Sharma, V. S. Rangra, and N. S. Negi, Z. Phys. Chem. 220, 325 (2006).

[4] R. Kumar, V. S. Rangra, D. R. Sharma, N. Thakur, and N. S. Negi, Z. Naturforsch. 61a, 197 (2006).

[5] S. Kumar, D. R. Sharma, N. Thakur, N. S. Negi, and V. S. Rangra, Z. Phys. Chem. 219, 1649 (2005).

[6] V.S. Rangra and D. R. Sharma, Indian J. Phys. B 78, 111 (2004).

[7] S. L. Abd-El-Messieh, J. Mol. Liq. 95, 167 (2002).

[8] A. D. Vyas and V. A. Rana, Indian J. Pure Appl. Phys. 40, 69 (2002).

[9] A. Chaudhari, S. Ahire, and S. C. Mehrotra, J. Mol. Liq. 94, 17 (2001). of the dipole moment of $\mathrm{C}_{6} \mathrm{H}_{5} \mathrm{CN}$ in benzene with increasing temperature may be attributed to a possible solute-solvent molecular association. The measured values of the energy parameters for the dielectric relaxation process and their comparison with the energy parameters for the viscous flow process show that the dielectric relaxation process may be treated as a rate process just like the viscous flow process.

[10] J. S. Dhull, D. R. Sharma, D. S. Gill, and K. N. Lakshminarayana, Indian J. Phys. B 56, 334 (1982).

[11] W. M. Heston (Jr.), A. D. Franklin, E. L. Hennely, and C. P. Smyth, J. Am. Chem. Soc. 72, 3443 (1950).

[12] K. V. Gopala Krishna, Trans. Farad. Soc. 53, 767 (1957).

[13] H. Eyring, S. Glasstone, and K. J. Laidler, Theory of Rate Process, McGraw-Hill, New York 1941, p. 541.

[14] N. E. Hill, W. E. Vaughan, A. H. Price, and M. Davies, Dielectric Properties and Molecular Behaviour, Van Nostrand-Reinhold, London 1969, p. 63.

[15] P. Debye, Polar Molecules, Chemical Catalog, New York 1929.

[16] A. Sharma and D. R. Sharma, J. Phys. Soc. Jpn. 61, 1049 (1992).

[17] F. H. Branin and C. P. Smyth, J. Chem. Phys. 42, 2503 (1965). 